\title{
The effect of low-sodium dialysate on ambulatory blood pressure measurement parameters in patients undergoing hemodialysis
}

This article was published in the following Dove Press journal:

Therapeutics and Clinical Risk Management

II December 2015

Number of times this article has been viewed

\author{
Serkan Akdag' \\ Aytac Akyol' \\ Huseyin Altug Cakmak ${ }^{2}$ \\ Aydin Rodi Tosu ${ }^{3}$ \\ Muntecep Asker' \\ Mehmet Yaman ${ }^{4}$ \\ Naci Babat' \\ Yasemin Soyoral ${ }^{5}$ \\ Muhammed Bilal Cegin ${ }^{6}$ \\ Ali Kemal Gur ${ }^{7}$ \\ Hasan Ali Gumrukcuoglu' \\ 'Department of Cardiology, Yuzuncu \\ Yil University Medical Faculty, Van, \\ ${ }^{2}$ Department of Cardiology, Rize \\ Kackar Government Hospital, \\ Rize, ${ }^{3}$ Department of Cardiology, \\ Mehmet Akif Ersoy Thoracic and \\ Cardiovascular Surgery Education \\ and Training Hospital, Istanbul, \\ ${ }^{4}$ Department of Cardiology, Samsun \\ Education and Training Hospital, \\ Samsun, ${ }^{5}$ Department of Nephrology, \\ ${ }^{6}$ Department of Anesthesiology \\ and Reanimation, ${ }^{7}$ Department of \\ Cardiovascular Surgery, Yuzuncu Yil \\ University Medical Faculty, Van, Turkey
}

Correspondence: Serkan Akdag Department of Cardiology, Yuzuncu Yil University Medical Faculty, Campus Street, Van 65100, Turkey

$\mathrm{Tel}+905546983126$

Fax +90 $432217 \quad 1717$

Email sercardiocon@hotmail.com.tr
Background: End stage renal disease is related to increased cardiovascular mortality and morbidity. Hypertension is an important risk factor for cardiovascular disorder among hemodialysis (HD) patients. The aim of this study was to investigate the effect of low-sodium dialysate on the systolic blood pressure (SBP) and diastolic blood pressure (DBP) levels detected by ambulatory BP monitoring (ABPM) and interdialytic weight gain (IDWG) in patients undergoing sustained HD treatment.

Patients and methods: The study included 46 patients who had creatinine clearance levels less than $10 \mathrm{~mL} / \mathrm{min} / 1.73 \mathrm{~m}^{2}$ and had been on chronic HD treatment for at least 1 year. After the enrollment stage, the patients were allocated low-sodium dialysate or standard sodium dialysate for 6 months via computer-generated randomization.

Results: Twenty-four hour SBP, daytime SBP, nighttime SBP, and nighttime DBP were significantly decreased in the low-sodium dialysate group $(P<0.05)$. No significant reduction was observed in both groups in terms of 24-hour DBP and daytime DBP $(P=\mathrm{NS})$. No difference was found in the standard sodium dialysate group in terms of ABPM. Furthermore, IDWG was found to be significantly decreased in the low-sodium dialysate group after 6 months $(P<0.001)$.

Conclusion: The study revealed that low-sodium dialysate leads to a decrease in ABPM parameters including 24-hour SBP, daytime SBP, nighttime SBP, and nighttime DBP and it also reduces the number of antihypertensive drugs used and IDWG.

Keywords: hypertension, sodium dialysate, hemodialysis

\section{Introduction}

End stage renal disease (ESRD) affects up to $15 \%$ of the adult population which is associated with increased cardiovascular complication. ${ }^{1,2}$ Hypertension (HT) can be present in up to $90 \%$ of people with ESRD. ${ }^{3}$ The prevalence of HT is higher in patients with ESRD, and it is the most important changeable risk factor for morbidity and mortality of patients with ESRD. ${ }^{4}$ Furthermore, HT is an independent risk factor for cardiovascular disease (CVD) among hemodialysis (HD) patients. ${ }^{5}$ It has been reported that the role of HT in the development of major adverse cardiovascular events applies equally to patients with HD as to the general population. ${ }^{5}$ Consequently, correct blood pressure (BP) measurement is a critical component of the treatment of people with ESRD both for risk reduction and for the suitable use of antihypertensive medication and lifestyle modification to management BP. ${ }^{6}$

Ambulatory BP monitoring (ABPM) is believed to be a better indicator of true BP measurements and variations than office BP, since this technique can obtain multiple BP readings in the ambulatory setting. ABPM has been reported to be a 
better predictor of target-organ damage and cardiovascular mortality and morbidity than office BP measurements in patients with $\mathrm{HT}^{7}{ }^{7}$ The recent studies have investigated the BP variations in patients with HD. ${ }^{8}$ While common increase was detected in systolic BP (SBP) and diastolic BP (DBP) in the early interdialytic period, the lack of normal nocturnal decline was also demonstrated. Although its use in patients with ESRD is compelling, ABPM has better reproducibility than office BP measurements. ${ }^{8}$

Since there is no information in the literature regarding the effect of low-sodium dialysate on SBP and DBP levels detected by ABPM and interdialytic weight gain (IDWG) in patients undergoing sustained HD treatment, we aimed to investigate this association in the present study.

\section{Patients and methods}

\section{Patient selection}

The present study was a single-center, randomized controlled and double-blinded trial. The patients enrolled in this study had a creatinine clearance less than $10 \mathrm{~mL} / \mathrm{min} / 1.73 \mathrm{~m}^{2}$ and had been on intermittent HD treatment for at least 1 year in a university hospital between March and December 2013. The exclusion criteria for the present study were masking or white coat HT, heart failure, cardiomyopathies, acute coronary syndromes, chronic ischemic heart disease, acute or chronic liver disease, endocrine or pulmonary diseases, valvular heart diseases, malignancies, active urinary tract infections, hemoglobin levels below $8 \mathrm{~g} / \mathrm{dL}$, and hypotension tendency. Sixty-four patients were enrolled for eligibility and after the initial assessment, 14 subjects were excluded from further analysis. Totally, 50 patients were included in the present study. The study was performed in compliance with the Declaration of Helsinki, and the study protocol was approved by the Yuzuncu Yil University, Faculty of Medicine Ethics Committee. Written informed consent was also obtained from the patients. The ClinicalTrials.gov identifier is NCT02621450. The patients were randomly divided into two equal groups as the low-sodium dialysate and the standard sodium dialysate groups. Three patients withdrew due to cramps or intradialytic hypotensive attacks from the low-sodium dialysate group and one patient withdrew from the standard sodium dialysate group. There were no other side effects in both dialysate groups. As a result, 46 patients (17 women and 29 men) were included into the study.

Clinical and laboratory profiles of the patients including cause of renal disease, history of HT, and antihypertensive treatments were recorded by systematic review of the patient files. After the enrollment stage, the patients were allocated

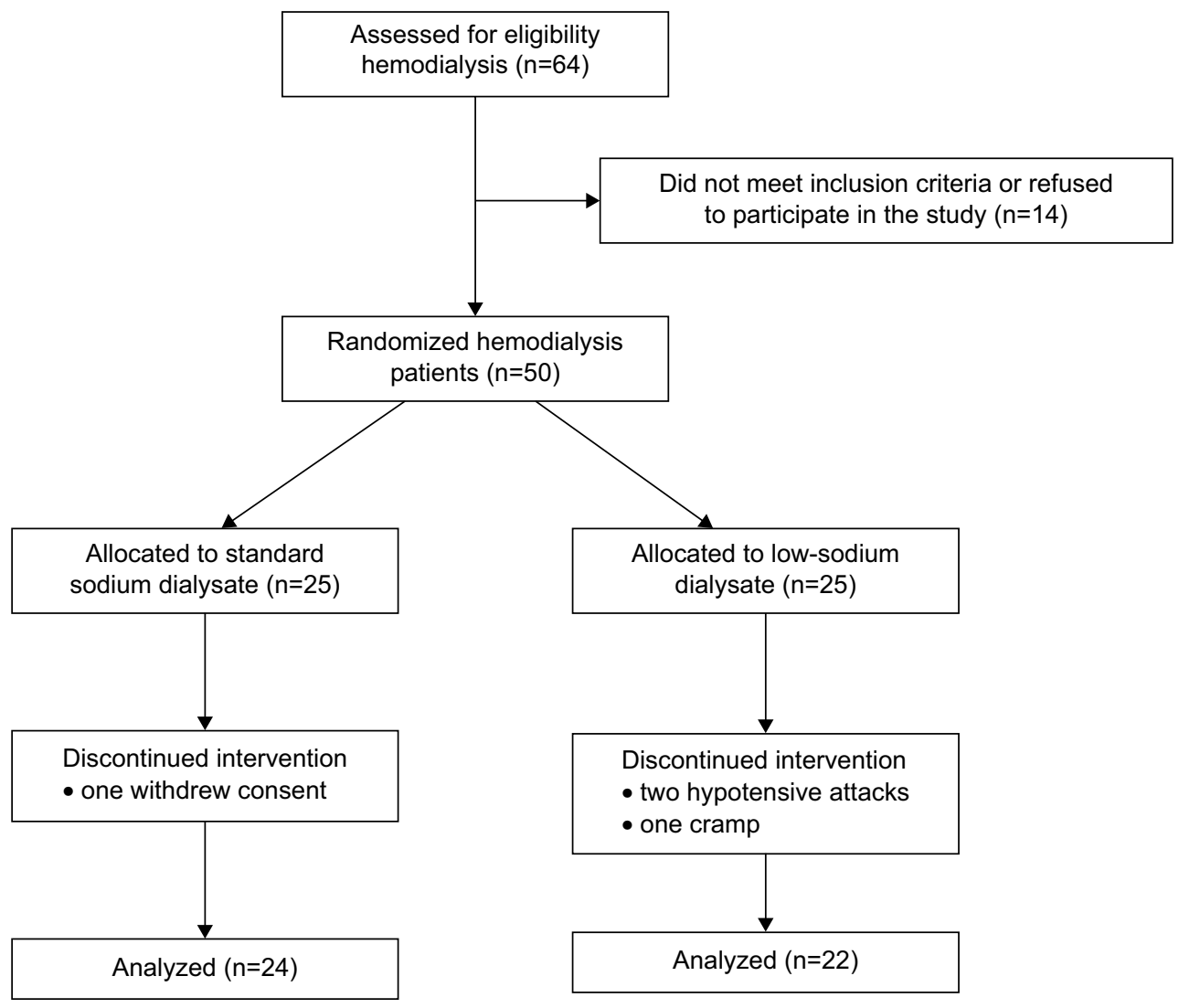

Figure I Selection of study participants and flow chart. 
low-sodium dialysate or standard sodium dialysate for 6 months via computer-generated randomization (Figure 1).

They were dialyzed three times a week with synthetic polysulfone (hollow-fiber, low flux, KUF $<20,1.6 \mathrm{~m}^{2}$ ) membrane, each session lasting 4 hours with bicarbonate dialysate (with $33 \mathrm{mmol} / \mathrm{L}$ concentration) and 300-350 $\mathrm{mL} / \mathrm{min}$ blood-flow. The ion content of the dialysate for all patients for the last 6 months was as follows: calcium of $1.5 \mathrm{mmol} / \mathrm{L}$, magnesium of $0.5 \mathrm{mmol} / \mathrm{L}$, and sodium of $140 \mathrm{mEq} / \mathrm{L}$.

\section{Study protocol}

All patients were assessed before and 6 months after the study. Before initiating the study, all patients were dialyzed with $140 \mathrm{mEq} / \mathrm{L}$ sodium concentration. In the low-sodium dialysate group, the dialysate sodium concentration was reduced from 140 to $137 \mathrm{mEq} / \mathrm{L}$. To eliminate the acute effects of HD on the parameters measured, baseline and end-of-study measurements were performed in nondialysis day, 24 hours after the midweek session.

IDWG was diagnosed as the change in body weight between two sequential HD sessions and was calculated by subtracting the dry weight from the weight before dialysis. Dialysis-related symptoms such as hypotensive attacks or cramps and requirement for saline infusion because of hypotensive attacks/cramps were monitored during the HD session. Hypotensive attack was defined as a reduction of $20 \mathrm{mmHg}$ in SBP associated with the symptoms requiring medical attention such as Trendelenburg position or saline infusion. Cramp was described as symptoms that required emergency medical attention or saline infusion without a reduction in BP. Saline infusion was defined as a $100 \mathrm{~mL}$ of saline solution $(0.9 \%)$, which was infused for 5 minutes to improve cramps or hypotensive attacks.

\section{Ambulatory BP measurement}

Ambulatory BP measurement during 24 hours was performed using a Space Labs 90207 oscillometric method (Spacelabs Healthcare, Inc., Redmond, WA, USA). The BP measurements were performed automatically from nonfistula arm. The confirmation of the device was checked with the standard auscultatory method in order to ensure that the changes in BP values between the two methods did not exceed $+5 \mathrm{mmHg}$.

The device was set to achieve the BP evaluation at 30 -minute intervals during the night ( $11 \mathrm{pm}$ to $7 \mathrm{am})$ and at 20 -minute intervals during the day ( 7 am to $11 \mathrm{pm}$ ). Each ABPM dataset was first automatically checked to remove erroneous readings. The data were checked by removing all readings of zero, heart rate readings out of 20-200 per minute,
DBP recordings out of $40-150 \mathrm{mmHg}, \mathrm{SBP}$ recordings out of 70-240 mmHg. All patients were educated to sleep or rest between $11 \mathrm{pm}$ and $7 \mathrm{am}$ (nighttime) and to continue their casual activities between 7 am and 11 pm (daytime). The patients had also been warned that they would need to keep the monitor on through the night - they could put the machine under the pillow or on the bed while you sleep. Because of variations in the number of ABPM measurements for each study participant, we could not identify a specific cutoff number of readings for inclusion criteria for our study. The reading numbers at night were enough to do appropriate statistical analyses.

"Nocturnal dipping" was defined as a decrease of greater than $10 \%$ (when compared with the daytime values) in the SBP or DBP at night. "Non-nocturnal dipping" was defined as a decrease of less than $10 \%$ (when compared with the daytime values) in the BP parameters levels at night or did not decrease in the SBP or DBP at night.

\section{Statistical analysis}

Continuous, normally distributed variables were expressed as mean $\pm \mathrm{SD}$. Categorical variables were expressed as frequencies and/or percentages. The Kolmogorov-Smirnov test was used to evaluate whether the continuous variables were normally distributed. Student's $t$-test was used for the comparison of normally distributed continuous numerical variables, the Mann-Whitney $U$-test was used for nonnormally distributed numerical variables, and the $\chi^{2}$-test was used for comparing categorical variables between the two groups. Since we have not encountered any loss of data or patient withdrew issue after randomization, we did not do intention-to-treat analysis. A two-sided $P$-value was considered for all comparisons. Statistical significance was defined as $P<0.05$. Statistical analyses were carried out using the Statistical Package for Social Sciences for Windows 13.0 (SPSS Inc., Chicago, IL, USA).

\section{Results}

The data of 46 patients were included in the statistical analyses at the end of the study (Figure 1). Baseline demographic, clinical, and hematological data of the patients are presented in Table 1. Mean HD time was 49.4 \pm 35.6 months. There was no difference between the two groups in terms of baseline demographic, clinical, and laboratory parameters.

Ambulatory BP measurements, IDWG and antihypertensive medications of the two groups at baseline and after 6 months were presented in Table 2. The most prescribed antihypertensive drug was calcium channel blockers, followed by angiotensin receptor blockers and beta blockers. 
Table I Comparison of demographic, clinical, and laboratory characteristics of the study population

\begin{tabular}{llll}
\hline & Low-sodium dialysate $(\mathbf{n}=\mathbf{2 2})$ & Standard sodium dialysate $(\mathbf{n}=\mathbf{2 4})$ & $\mathbf{P}$-value \\
\hline Age (years) & $45.2 \pm 2.8$ & $43.6 \pm 2.6$ & $\mathrm{NS}$ \\
Sex (male/female) & $13 / 9$ & $14 / 10$ & $\mathrm{NS}$ \\
Dialysis duration (months) & $52.1 \pm 37.9$ & $55.6 \pm 32.3$ & $\mathrm{NS}$ \\
Urea reduction rate (\%) & $70.4 \pm 4.5$ & $68.9 \pm 3.8$ & $\mathrm{NS}$ \\
Creatinine (mg/dL) & $7.2 \pm 3.4$ & $7.1 \pm 3.2$ & $\mathrm{NS}$ \\
Sodium (mEq/L) & $138 \pm 4.1$ & $139 \pm 3.8$ & $\mathrm{NS}$ \\
Calcium (mg/dL) & $9.1 \pm 1.2$ & $9.2 \pm 1.3$ & $\mathrm{NS}$ \\
Phosphorus (mg/dL) & $5.3 \pm 1.6$ & $5.2 \pm 1.5$ & $\mathrm{NS}$ \\
Albumin (mg/dL) & $3.9 \pm 0.5$ & $3.9 \pm 0.4$ & $\mathrm{NS}$ \\
Hemoglobin (g/dL) & $9.8 \pm 0.6$ & $9.7 \pm 0.7$ & $\mathrm{NS}$ \\
Diabetes mellitus, $\mathrm{n}(\%)$ & $6(27.3)$ & $7(29.2)$ & $\mathrm{NS}$ \\
Hypertension, $\mathrm{n}(\%)$ & $13(59.1)$ & $15(62.5)$ & $\mathrm{NS}$ \\
\hline
\end{tabular}

Note: Values are presented as mean \pm standard deviation or number (\%), as appropriate.

Abbreviation: NS, nonsignificant.

At the baseline, a total of 28 patients $(60.8 \%)(13$ [59.1\%] in the low sodium group vs 15 [62.5\%] in the standard sodium group) had used antihypertensive drug. The mean number of antihypertensive drugs of the study patients was

Table 2 Baseline and 6 months ambulatory blood pressure measurements, interdialytic weight gain and number of antihypertensive medications

\begin{tabular}{|c|c|c|c|}
\hline & Baseline & 6 months & $\overline{P \text {-value }}$ \\
\hline \multicolumn{4}{|l|}{ 24-hour SBP (mmHg) } \\
\hline Low-sodium dialysate $(n=22)$ & $136 \pm 22$ & $127 \pm 19$ & $<0.05$ \\
\hline Standard sodium dialysate $(n=24)$ & $135 \pm 18$ & $130 \pm 14$ & NS \\
\hline \multicolumn{4}{|l|}{ 24-hour DBP (mmHg) } \\
\hline Low-sodium dialysate $(n=22)$ & $73 \pm 10$ & $69 \pm 10$ & NS \\
\hline $\begin{array}{l}\text { Standard sodium dialysate }(\mathrm{n}=24) \\
\text { Daytime SBP }(\mathrm{mmHg})\end{array}$ & $73 \pm 9$ & $7 I \pm 8$ & NS \\
\hline Low-sodium dialysate $(\mathrm{n}=22)$ & $138 \pm 22$ & $130 \pm 19$ & $<0.05$ \\
\hline Standard sodium dialysate $(n=24)$ & $137 \pm 18$ & $133 \pm 15$ & NS \\
\hline \multicolumn{4}{|l|}{ Daytime DBP $(\mathrm{mmHg})$} \\
\hline Low-sodium dialysate $(\mathrm{n}=22)$ & $75 \pm 11$ & $7 I \pm 11$ & NS \\
\hline Standard sodium d & $74 \pm 9$ & $72 \pm 8$ & NS \\
\hline \multicolumn{4}{|l|}{ Nighttime SBP $(\mathrm{mmHg})$} \\
\hline Low-sodium dialysate $(\mathrm{n}=22)$ & $13 \mid \pm 26$ & $120 \pm 27$ & $<0.05$ \\
\hline Standard sodium dialys & $129 \pm 19$ & $125 \pm 17$ & NS \\
\hline \multicolumn{4}{|l|}{ Nighttime DBP (mmHg) } \\
\hline Low-sodium dialysate $(n=22)$ & $69 \pm 11$ & $64 \pm 8$ & $<0.05$ \\
\hline Standard sodium dialysate $(n=$ & $69 \pm 9$ & $68 \pm 11$ & NS \\
\hline \multicolumn{4}{|l|}{ Antihypertensive medication } \\
\hline Low-sodium dialysate $(\mathrm{n}=22)$ & $2.1 \pm 0.8$ & $1.2 \pm 0.4$ & $<0.00 \mathrm{I}$ \\
\hline Standard sodium dialysate $(n=24)$ & $1.8 \pm 0.7$ & $1.7 \pm 0.8$ & NS \\
\hline \multicolumn{4}{|l|}{ IDWG (gr) } \\
\hline Low-sodium dialysate $(\mathrm{n}=22)$ & $2,256 \pm 939$ & $1,648 \pm 589$ & $<0.00 \mathrm{I}$ \\
\hline Standard sodium dialysate $(n=24)$ & $2,384 \pm 8 I I$ & $2,147 \pm 762$ & NS \\
\hline \multicolumn{4}{|l|}{ Nocturnal non-dipping } \\
\hline Low-sodium dialysate $(n=22)$ & $12(54.5 \%)$ & $9(40.9 \%)$ & NS \\
\hline Standard sodium dialysate $(n=24)$ & II (45.8\%) & $10(41.7 \%)$ & NS \\
\hline
\end{tabular}

Notes: Data is presented as mean \pm standard deviation or number (\%), as appropriate. NS refers to normal statistic $(P>0.05)$.

Abbreviations: DBP, diastolic blood pressure; IDWG, interdialytic weight gain; SBP, systolic blood pressure.
2.1 in the low sodium group and 1.8 in the standard sodium group respectively at baseline. Moreover, after 6-month follow-up, mean number of antihypertensive drugs significantly decreased to 1.2 in the low sodium group and to 1.7 in the standard sodium group.

Twenty-four hour SBP, daytime SBP, nighttime SBP, and nighttime DBP were significantly decreased in the lowsodium dialysate group $(P<0.05)$. There was no significant reduction in two groups in terms of 24-hour DBP and daytime DBP. Furthermore, there was no difference in the standard sodium dialysate group regarding ABPM. Furthermore, IDWG was found to be significantly decreased in the lowsodium dialysate group compared to the standard group at the end of the study $(P<0.001)$.

\section{Discussion}

The present study yielded two major results: first, 24-hour SBP, daytime SBP, and nighttime SBP, and DBP were reduced in the low-sodium dialysate group, and second, average antihypertensive drug use and IDWG were decreased significantly in the low-sodium dialysate group.

CVD is a leading cause of mortality accounting for $30 \%-50 \%$ of all deaths in patients with ESRD. ${ }^{9} \mathrm{HT}$, which coexists in up to $90 \%$ of people with ESRD is a major risk factor for cardiovascular morbidity and mortality in patients undergoing HD therapy. ${ }^{3}$

The pathogenesis of HT in patients with ESRD is multifactorial and includes volume retention and deterioration and endothelial dysfunction. ${ }^{2}$ In this population, HT may be primarily caused by excess extracellular fluid volume. ${ }^{10}$ The total sum of sodium in the body controls the extracellular volume. In ESRD, sodium equation becomes positive and the extracellular volume becomes enlarged, causing 
endothelial impairment and HT. ${ }^{11}$ Dishy et al ${ }^{12}$ showed that the endothelium is directly affected by extracellular sodium amount, which is independent from BP levels. Human studies have shown that endothelial function is improved by limited sodium intake and impaired by high dietary sodium. ${ }^{12,13}$ In patients with HD, sodium intake is determined not only by diet but also by the sodium content of the dialysate. ${ }^{14}$ The aim of this study was to investigate the effect of low-sodium dialysate on SBP and DBP levels detected by ABPM and IDWG in patients undergoing sustained HD treatment.

Because of inconstancy in BP and different techniques of measuring BP, the diagnosis of HT is difficult in patients with ESRD. ${ }^{15}$ Use of a single-office BP evaluation may overdiagnose HT in the general population up to $30 \%$, which is described as "white-coat HT". ${ }^{16}$ Conversely, some objects with a normal office BP value have a high ambulatory BP, described as "masked HT". Andersen et $\mathrm{al}^{17}$ and Bangash and Agarwal ${ }^{18}$ reported that the prevalence of "masked HT" as $33 \%$ in patients. Therefore, the use of a single-clinic BP value may lead to insufficient treatment in high-risk patients. ${ }^{19}$ In addition to the shortfalls of a single BP measurement, inaccuracies may be caused by operator errors, which include inappropriate cuff usage, inadequate rest period, incorrect positioning, and overly rapid deflation rate. ${ }^{19}$

ABPM is a technique that may decrease the measured $\mathrm{BP}$ variability and is an important tool for clarifying the mean level of BP, nocturnal HT, and the non-dipping phenomenon. ${ }^{20}$ ABPM permits BP to be measured over extended period and is considered as the gold standard for the determination of BP levels. ${ }^{21}$ An additional gain is the identification of "non-dipping" patients, which is very common in patients with ESRD and may be a significant determinant for increased cardiovascular complication. ${ }^{22,23}$ In our study, there were 23 (50\%) non-dipping patients, which is higher than the study by Liu et $\mathrm{al}^{24}$ and Ekart et al, ${ }^{25}$ in which $30 \%$ and $31.5 \%$ patients with HD were dippers, respectively. Also in our study, no significant reduction was found in terms of the rate of non-dipping between baseline and 6-month follow-up.

The National Kidney Foundation Kidney Disease Outcomes Quality Initiative guidelines suggest that the pre- and post-HD BP should be $<140 / 90$ and $<130 / 80 \mathrm{mmHg}$, respectively. ${ }^{26}$ Compared to pre- or postdialysis BP measurements, ambulatory BP correlates better with all-cause mortality. ${ }^{27}$ The 2013 guidelines from the European Society of Cardiology/European Society of Hypertension for the clinical management of primary HT in adults recommend that in those patients the results of home BP monitoring for diagnosis of
$\mathrm{HT}$ are $\mathrm{SBP} / \mathrm{DBP}>130 / 80$ at 24 hours, $\mathrm{SBP} / \mathrm{DBP}>135 / 85$ at daytime, and SBP/DBP $>120 / 70$ nighttime. ${ }^{28}$ In our study, the lowering of the dialysate sodium in the dialysis patients led to considerable improvement in ABPM parameters including 24-hour SBP, daytime SBP, nighttime SBP, and nighttime DBP compared with the standard dialysate group (Table 2). Additionally, decreasing the dialysate sodium concentration from 140 to $137 \mathrm{mEq} / \mathrm{L}$ led to a decrease in the number of antihypertensive drugs used for treatment $(2.1 \pm 0.8$ vs $1.2 \pm 0.4, P<0.001)$.

The second interesting finding of the present study is that a considerable improvement was achieved in IDWG in the low-sodium dialysate group. Kalantar-Zadeh et $\mathrm{al}^{29}$ showed that higher IDWG is associated with increased cardiovascular mortality rate in patients with ESRD. The main cause of IDWG between two dialysis sessions is the water and salt intake. A lowering of the dialysate sodium concentration has been shown to provide more effective sodium reduction that may also reduce IDWG and thirst. ${ }^{30} \mathrm{We}$ demonstrated that lowering dialysate sodium from $140 \mathrm{mEq} / \mathrm{L}$ to $137 \mathrm{mEq} / \mathrm{L}$ recovered the BP parameters as well as the mean IDWG at the end of the study. We consider that the abstention of excessive sodium taking during HD may improve IDWG and $\mathrm{BP}$ in patients with ESRD patients. Sodium concentration of dialysate is also related to improved BP control. Several previous studies showed that the reduction of sodium amount of dialysate will cause improvement in BP. ${ }^{31,32}$ Davenport reported that higher dialysate sodium contents indicate greater numbers of antihypertensive drugs to regulate BP in patients with ESRD. ${ }^{33}$

A high-salt dietary intake has been implicated in CVD, glucose intolerance, autoimmune conditions, and cancer. A high-salt diet impairs treatment responses to antihypertensive drugs and hence it is presumed to exacerbate heart failure. Although a link between a high-salt intake and increased BP appears irrefutable, the precise mechanisms remain elusive. Since the 24-hour sodium excretion is similar to the patient's intake and sodium ions resides extracellularly, the salt content of the body remains steady after a few days when the salt intake is changed. The sodium concentration is similar in plasma and interstitium, thereby creating an isotonic microenvironment. Hence, sodium in the human body and volume regulation are closely coupled. In a recent study, Titze et al observed that salt is also stored largely nonosmotically bound to glycosaminoglycans in the skin interstitium and connective tissue. ${ }^{34,35}$ The storage is dynamic and tightly regulated by monocyte phagocytic system cells. ${ }^{36}$ The 
resulting skin mircoenvironment is hypertonic to plasma and is sensed by monocyte phagocytic system cells through tonicity-responsive enhancer-binding protein (TonEBP) that signals vascular endothelial growth factor (VEGF)-C production. VEGF-C in turn causes an increase in lymphcapillary density that facilitates the clearance of salt from skin stores. The absence of TonEBP or VEGF-C, blockade of VEGF-C receptor, or increased expression of the soluble form of the VEGF-C receptor, results in faulty salt clearance from skin and salt-sensitive HT. ${ }^{37}$ It may speculate that the potential reduction in extravascular salt stores like the skin and connective tissue with lower sodium dialysate protocol according to Titze et al's study. ${ }^{34,35}$

Potassium excretion decreases and often seizes with advanced renal failure and to maintain proper balance, this ion has to be removed with by HD. The standard dialysate potassium level in most dialysis units is $2 \mathrm{mEq} / \mathrm{L}$. With the use of such a level, the serum potassium value at the end of a dialysis session is often fairly low. This is because, during dialysis, the removed potassium comes mainly from the relatively small extracellular compartment, thus leading to the immediate postdialysis serum level to be low even though the amount removed is not that substantial. In this regard, the average amount removed by a dialysis treatment is in the realm of $50 \mathrm{mEq} / \mathrm{L} .{ }^{38}$ However, after a few hours, the serum potassium concentration would bounce back to a value not too far from the pre-dialysis level as a result of the entry of intracellular potassium into the blood. Since the serum potassium level can be quite low for a short period during dialysis, hemodynamically significant arrhythmia may develop due to temporary hypokalemia. This problem can be addressed by increasing dialysate potassium concentration. This hypokalemia-induced arrhythmogenic effect is potentiated by the concomitant administration of digitalis-related preparations. In our study, if the patient was not given the digital-related preparations and potassium level was not $<4.5 \mathrm{mEq} / \mathrm{L}$ in pre-dialysis period, the target potassium level was $2 \mathrm{mEq} / \mathrm{L}$. Otherwise, the target potassium level was $3 \mathrm{mEq} / \mathrm{L}$.

On the other hand, Ozturk et $\mathrm{al}^{39}$ reported that lowered sodium content of dialysate from $140 \mathrm{mEq} / \mathrm{L}$ to $135 \mathrm{mEq} / \mathrm{L}$ led to a remarkable increase in hypotensive attacks and in the frequency of cramps. In our study, low-dialysate sodium was associated with worsening dialysis-related symptoms and three patients withdrew due to cramps associated with low sodium or intradialytic hypotensive attacks. Therefore, careful attention to dialysate sodium prescription is essential to optimize BP levels, IDWG, and intradialytic cramps or symptoms.

\section{Study limitations}

There are some limitations associated with the present study. First, this study had a relatively small study population and arose from a single-center. Secondly, the study provides no information regarding short- or long-term clinical outcomes in patients with ESRD undergoing HD treatment.

\section{Conclusion}

In conclusion, our study revealed that low-sodium dialysate leads to a decrease in ABPM parameters including 24-hour SBP, daytime SBP, nighttime SBP, and nighttime DBP and it also reduces the number of antihypertensive drugs used and IDWG. Further large-scale randomized placebo controlled studies are needed to determine the safety and possible detrimental effects of this procedure among patients with HD.

\section{Disclosure}

The authors report no conflicts of interest in this work.

\section{References}

1. Coresh J, Astor BC, Greene T, Eknoyan G, Levey AS. Prevalence of chronic kidney disease and decreased kidney function in the adult US population: Third National Health and Nutrition Examination Survey. Am J Kidney Dis. 2003;41:1-12.

2. Gansevoort RT, Matsushita K, van der Velde M, et al. The Chronic Kidney Disease Prognosis Consortium: lower estimated GFR and higher albuminuria are associated with adverse kidney outcomes: a collaborative meta-analysis of general and high-risk population cohorts. Kidney Int. 2011;80:93-104.

3. Sarafidis PA, Li S, Chen SC. Hypertension awareness, treatment, and control in chronic kidney disease. Am J Med. 2008;121:332-340.

4. Mogensen CE. The kidney in diabetes: how to control renal and related cardiovascular complications. Am J Kidney Dis. 2001;37(Suppl 2):S2-S6.

5. Port FK, Hulbert-Shearon TE, Wolfe RA. Predialysis blood pressure and mortality risk in a national sample of maintenance hemodialysis patients. Am J Kidney Dis. 1999;33:507-517.

6. National Institute for Health and Clinical Excellence (NICE). Chronic Kidney Disease: Early Identification and Management of Chronic Kidney Disease in Adults in Primary and Secondary Care. London: National Institute for Health and Care Excellence; 2008.

7. Staessen JA, Thijs L, Fagard R, et al. Predicting cardiovascular risk using conventional vs ambulatory blood pressure in older patients with systolic hypertension. Systolic Hypertension in Europe Trial Investigators. JAMA. 1999;282:539-546.

8. Mansoor GA, McCabe E, White WB. Long-term reproducibility of ambulatory blood pressure. J Hypertens. 1994;12:703-708.

9. Levey AS, Beto JA, Coronado BE, et al. Controlling the epidemic of cardiovascular disease in chronic renal disease: what do we know? what do we need to learn? Where do we go from here? National foundation task force on cardiovascular disease. Am J Kidney Dis. 1998;32:853-906.

10. Fishbane S, Natke E, Maesaka JK. Role of volume overload in dialysisrefractory hypertension. Am J Kidney Dis. 1996;28:257-261.

11. Charra B. Fluid balance, dry weight, and blood pressure in dialysis. Hemodial Int. 2007;11:21-31.

12. Dishy V, Sofowora GG, Imamura H, et al. Nitric oxide production decreases after salt loading but is not related to blood pressure changes or nitric oxide-mediated vascular responses. J Hypertens. 2003;21: $153-157$. 
13. Matrougui K, Lévy BI, Schiavi P, Guez D, Henrion D. Indapamide improves flow induced dilation in hypertensive rats with a high salt intake. J Hypertens. 1998;16:1485-1490.

14. Van Stone JC, Bauer J, Carey J. The effect of dialysate sodium concentration on body fluid compartment volume, plasma renin activity and plasma aldosterone concentration in chronic hemodialysis patients. Am J Kidney Dis. 1982;2:58-64.

15. Agarwal R, Nissenson AR, Batlle D, Coyne DW, Trout JR, Warnock DG. Prevalence, treatment, and control of hypertension in chronic hemodialysis patients in the United States. Am J Med. 2003;115:291-297.

16. Agarwal R, Anderson MJ. Prognostic importance of clinic and home blood pressure recordings in patients with chronic kidney disease. Kidney Int. 2006;69:406-411.

17. Andersen MJ, Khawandi W, Agarwal R. Home blood pressure monitoring in CKD. Am J Kidney Dis. 2005;45:994-1001.

18. Bangash F, Agarwal R. Masked hypertension and white-coat hypertension in chronic kidney disease: a meta-analysis. Clin J Am Soc Nephrol. 2009;4:656-664.

19. McAlister FA, Straus SE. Evidence based treatment of hypertension. Measurement of blood pressure: an evidence based review. BMJ. 2001;322:908-911.

20. Agarwal R, Peixoto AJ, Santos SFF, Zoccali C. Out-of-office blood pressure monitoring in chronic kidney disease. Blood Press Monit. 2009; 14:2-11.

21. Lovibond K, Jowett S, Barton P, et al. Cost-effectiveness of options for the diagnosis of high blood pressure in primary care: a modelling study. Lancet. 2011;378:1219-1230.

22. Mojón A, Ayala DE, Piñeiro L, et al. Comparison of ambulatory blood pressure parameters of hypertensive patients with and without chronic kidney disease. Chronobiol Int. 2013;30:145-158.

23. Brotman DJ, Davidson MB, Boumitri M, Vidt DG. Impaired diurnal blood pressure variation and all-cause mortality. Am J Hypertens. 2008;21:92-97.

24. Liu M, Takahashi H, Morita Y, et al. Non-dipping is a potent predictor of cardiovascular mortality and is associated with autonomic dysfunction in hemodialysis patients. Nephrol Dial Transplant. 2003;18: 563-569.

25. Ekart R, Kanič V, Pečovnik Balon B, Bevc S, Hojs R. Prognostic value of 48-hour ambulatory blood pressure measurement and cardiovascular mortality in hemodialysis patients. Kidney Blood Press Res. 2012; 35:326-331.

26. K/DOQI Workgroup. K/DOQI clinical practice guidelines for cardiovascular disease in dialysis patients. Am J Kidney Dis. 2005;45: S1-S153.
27. Alborzi P, Patel N, Agarwal R. Home blood pressures are of greater prognostic value than hemodialysis unit recordings. Clin J Am Soc Nephrol. 2007;2:1228-1234.

28. Mancia G, Fagard R, Narkiewicz K, et al. 2013 ESH/ESC practice guidelines for the management of arterial hypertension. Blood Press. 2014;23:3-16

29. Kalantar-Zadeh K, Regidor DL, Kovesdy CP, et al. Fluid retention is associated with cardiovascular mortality in patients undergoing longterm hemodialysis. Circulation. 2009;119:671-679.

30. Locatelli F, di Filippo S, Manzoni C. Relevance of the conductivity kinetic model in the control of sodium pool. Kidney Int. 2000;58: 89-95.

31. Gümrükçüoğlu HA, Arı E, Akyol A, et al. Effects of lowering dialysate sodium on carotid artery atherosclerosis and endothelial dysfunction in maintenance hemodialysis patients. Int Urol Nephrol. 2012; 44:1833-1839.

32. Farmer CKT, Donohoe P, Dallyn PE, et al. Low-sodium hemodialysis without fluid removal improves blood pressure control in hemodialysis patients. Nephrology. 2000;5:237-241.

33. Davenport A. Audit of the effect of dialysate sodium concentration on inter-dialytic weight gains and blood pressure control in chronic hemodialysis patients. Nephron Clin Pract. 2006;104:120-125.

34. Titze J, Müller DN, Luft FC. Taking another "look" at sodium. Can J Cardiol. 2014;30:473-475. doi:10.1016/j.cjca.2014.02.006.

35. Titze J, Shakibaei M, Schafflhuber M, et al. Glycosaminoglycan polymerization may enable osmotically inactive $\mathrm{Na}+$ storage in the skin. Am J Physiol Heart Circ Physiol. 2004;287:H203-H208.

36. Machnik A, Neuhofer W, Jantsch J, et al. Macrophages regulate salt dependent volume and blood pressure by a vascular endothelial growth factor-c-dependent buffering mechanism. Nat Med. 2009;15: 545-552.

37. Wiig H, Schroder A, Neuhofer W, et al. Immune cells control skin lymphatic electrolyte homeostasis and blood pressure. J Clin Invest. 2013; 123:2803-2815.

38. Hou S, McElroy PA, Nootens J, Beach M. Safety and efficacy of lowpotassium dialysate. See comment in PubMed Commons below. Am J Kidney Dis. 1989;13:137-143.

39. Ozturk S, Taymez DG, Bahat G, et al. The influence of low dialysate sodium and glucose concentration on volume distributions in body compartments after hemodialysis: a bio impedance analysis study. Nephrol Dial Transplant. 2008;23:3629-3634.
Therapeutics and Clinical Risk Management

\section{Publish your work in this journal}

Therapeutics and Clinical Risk Management is an international, peerreviewed journal of clinical therapeutics and risk management, focusing on concise rapid reporting of clinical studies in all therapeutic areas, outcomes, safety, and programs for the effective, safe, and sustained use of medicines. This journal is indexed on PubMed Central, CAS,

\section{Dovepress}

EMBase, Scopus and the Elsevier Bibliographic databases. The manuscript management system is completely online and includes a very quick and fair peer-review system, which is all easy to use. Visit http://www.dovepress.com/testimonials.php to read real quotes from published authors. 